\title{
Analysis of Pvama1 genes from China-Myanmar border reveals little regional genetic differentiation of Plasmodium vivax populations
}

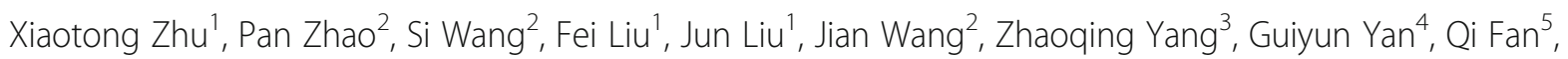
Yaming Cao ${ }^{1 *}$ and Liwang Cui ${ }^{1,6^{*}}$

\begin{abstract}
Background: With the premise of diminishing parasite genetic diversity following the reduction of malaria incidence, the analysis of polymorphic antigenic markers may provide important information about the impact of malaria control on local parasite populations. Here we evaluated the genetic diversity of Plasmodium vivax apical membrane antigen 1 (Pvama1) gene in a parasite population from the China-Myanmar border and compared it with global P. vivax populations.

Methods: We performed evolutionary analysis to examine the genetic diversity, natural selection, and population differentiation of 73 Pvama1 sequences acquired from the China-Myanmar border as well as 615 publically available Pvama1 sequences from seven global $P$. vivax populations.

Results: A total of 308 Pvama 1 haplotypes were identified among the global P. vivax isolates. The overall nucleotide diversity of Pvamal gene among the 73 China-Myanmar border parasite isolates was 0.008 with 41 haplotypes being identified $(H d=0.958)$. Domain I (DI) harbored the majority $(26 / 33)$ of the polymorphic sites. The McDonald Kreitman test showed a significant positive selection across the ectodomain and the DI of Pvamar. The fixation index ( $\left.F_{S T}\right)$ estimation between the China-Myanmar border, Thailand (0.01) and Myanmar (0.10) showed only slight geographical genetic differentiation. Notably, the Sal-I haplotype was not detected in any of the analyzed global isolates, whereas the Belem strain was restricted to the Thai population. The detected mutations are mapped outside the overlapped region of the predicted B-cell epitopes and intrinsically unstructured/disordered regions.
\end{abstract}

Conclusions: This study revealed high levels of genetic diversity of Pvama1 in the P. vivax parasite population from the China-Myanmar border with DI displaying stronger diversifying selection than other domains. There were low levels of population subdivision among parasite populations from the Greater Mekong Subregion.

Keyword: Plasmodium vivax, Pvama1, Genetic diversity, China-Myanmar border, Malaria

\section{Background}

In South and Southeast Asia, Latin America and Oceania, Plasmodium vivax is the major malaria species; around 2.5 billion people are living in areas of $P$. vivax transmission [1]. In the Greater Mekong Subregion (GMS) where countries are pursuing regional malaria elimination

\footnotetext{
* Correspondence: ymcao@mail.cmu.edu.cn; luc2@psu.edu

'Department of Immunology, College of Basic Medical Science, China

Medical University, Shenyang, Liaoning 110122, China

Full list of author information is available at the end of the article
}

(www.apmen.org), $P$. vivax is often sympatric with $P$. falciparum, $P$. ovale and $P$. malariae [2], although $P$. vivax has become the predominant species in recent years [3]. With the ability to form hypnozoites that are responsible for relapses, $P$. vivax presents a major challenge for malaria elimination. Within the GMS, malaria is distributed very unevenly; malaria transmission is concentrated along international borders, whereas central plains are mostly free from malaria $[3,4]$. The more intensified control efforts in this region have led to a further reduction of 
malaria incidence, creating isolated areas or pockets of high malaria prevalence separated by areas with extremely low endemicity or malaria-free zones. In China, autochthonous malaria incidence is mostly located in counties bordering with Myanmar, where malaria burden is the highest in the GMS $[5,6]$. In these border regions, crossborder human migration as a major source of malaria introduction presents a significant challenge to the malaria elimination course $[7,8]$. Since control efforts are expected to have great impacts on the genetic diversity of the parasite populations $[9,10]$, tracking their spatial and temporal dynamics may provide timely measurement of the progress of regional malaria elimination.

The genetic diversity of antigens in malaria parasites has been extensively studied not only because of their importance as malaria vaccine candidates [11], but also due to their usefulness as molecular markers for differentiating parasite populations. Several $P$. vivax proteins, including Duffy-binding protein (DBP), apical membrane antigen 1 (AMA1), and merozoite surface proteins (MSPs), have been selected as vaccine candidates for their essential functions during erythrocytes invasion and their antigenicity in natural host immune response [12-15]. Among them, AMA1 has been identified as an essential target of the host immune system, and considered an attractive malaria vaccine candidate [15-17]. The ama1 gene has been extensively studied in a number of Plasmodium species [18]. As a type I transmembrane protein, AMA1 is secreted by microneme organelles. Together with RON proteins, AMA1 is involved in merozoite reorientation and tight junction formation during the invasion process [19-24]. Antibodies raised against the AMA1 ectodomain have been shown to inhibit erythrocyte invasion, and AMA1 immunization protects against malaria infection [15, 25-27]. The ectodomain of AMA1 was divided into three subdomains referred to as Domain I (DI), Domain II (DII) and Domain III (DIII) based on the conserved cysteine residues [28]. DI harbors higher levels of genetic variation compared to DII and DIII, suggesting this domain is a target of the host immune system [29]. Within DI of PfAMA1, eight polymorphic amino acids located in the cluster 1 loop (c1L) were identified as the targets of allele-specific, protective immune response [30]. Evidence of diversifying selection was observed in DII of AMA1 in some studies such as in the Sri Lankan parasites, suggesting that this region may also be targeted by host immunity [31, 32]. In addition, serological studies showed that DII is the most immunogenic of the three domains [33]. Due to the highly polymorphic feature of the ama1 gene, it has been used as a molecular marker for population genetic studies [34, 35].

Although the genetic diversity of $P$. vivax ama1 (Pvama1) has been extensively studied with samples from Asia, Oceania and South America [36-39], there is a relative lack of data from Southeast Asia, where parasite populations are now being restricted to the international borders as the regional malaria elimination program is unfolding. Thus, in this study, we aimed to investigate the genetic diversity of Pvama1 gene from the ChinaMyanmar border area. We wanted to determine whether this potentially isolated parasite population was genetically different from those of other endemic regions. Furthermore, we wanted to learn whether the intensified malaria control efforts resulted in evident reduction of genetic diversity of the parasite population as observed elsewhere in Southeast Asia [9]. Our study revealed a considerably high level of genetic diversity of the Pvamal gene in the China-Myanmar border parasite population.

\section{Methods}

\section{Study sites and isolates}

Parasite sample collection was carried out from April 2011 to October 2012 around the township of Laiza, which is located in the northeast Kachin State, along the China-Myanmar border [40]. This region has a subtropical climate with most of the precipitation occurring in June-August (the rainy season). The majority of the local inhabitants are ethnic Kachin (called Jingpo in China) with farming being their major occupation. In recent years, there has been a considerable decline of annual malaria incidence and $P$. vivax has become the predominant parasite species [40, 41]. Like other international borders within the GMS, the China-Myanmar border also has experienced an increase in cross-border migration with large migrant populations engaging in international trade, logging, quarry, plantation and construction activities, which may play an important role for introducing malaria $[8,42]$. Filter paper blood spots were collected as our passive malaria case detection efforts in the township hospital and clinics servicing surrounding villages and two camps for internally displaced people. P. vivax infections were based on microscopy of Giemsa-stained thick smears. The study protocol was approved by the IRB of Pennsylvania State University and by the Health Department of Kachin.

\section{DNA extraction, amplification and sequencing of the PvAMA1 gene}

Genomic DNA from dried filter paper blood spots of slide-positive samples was extracted by using a QIAamp DNA Blood Mini kit (QIAGEN, Hilden, Germany). A DNA fragment of the Pvama1 gene encoding the extracellular region (1,290 bp, nucleotide region (nt) 148-1,437 of the Sal-I sequence; GenBank accession no. AF063138) was amplified and sequenced with six oligonucleotide primers listed in Additional file 1: Table S1. The amplifications were performed in a $20 \mu \mathrm{l}$ volume reaction containing $1 \times$ KOD-Plus-Neo buffer, $200 \mu \mathrm{M}$ dNTPs, $1 \mathrm{mM} \mathrm{MgSO}_{4}, 250$ $\mathrm{nM}$ of each primer, 0.4 units of KOD Plus neo polymerase 
(Toyobo, Osaka, Japan), and $1 \mu$ genomic DNA as template. The reaction was run at $94{ }^{\circ} \mathrm{C}$ for $5 \mathrm{~min}$, followed by 45 cycles of $94{ }^{\circ} \mathrm{C}$ for $15 \mathrm{~s}, 56^{\circ} \mathrm{C}$ for $15 \mathrm{~s}$, and $68{ }^{\circ} \mathrm{C}$ for $90 \mathrm{~s}$, and extension at $68{ }^{\circ} \mathrm{C}$ for $5 \mathrm{~min}$. PCR fragments were analyzed by electrophoresis on a $1.2 \%$ agarose gels. Direct sequencing of purified PCR fragments was carried out on both strands with primers listed in Additional file 1: Table S1 by using the ABI BigDye ${ }^{\mathrm{Tm}}$ Terminator Reaction Ready kit (Applied Biosystems, CA, USA).

\section{Sequence assembling and polymorphism analysis}

Of the $78 P$. vivax infections amplified by PCR, five samples showing dual peaks suggestive of a mixed infection were excluded from further analysis. Pvama1 was successfully sequenced from 73 samples (sequences submitted to GenBank under accession nos. KX495505-KX495577). A single contiguous 1,290 bp of Pvama1 (nt 148-1,437 and codons 50-479) was derived for each of the 73 Pvama1 sequences, which include DI (codons 94-247), DII (codons 265-363) and DIII (codons 388-451). The consensus of the sequences were aligned to the P. vivax Sal-I strain by using the CLUSTAL W program in MEGA6.0 [43]. Seven primate-adapted P. vivax isolates (Belem, Chesson I, India VII, Indonesia XIX, North Korea, Palo Alto and Simium; GenBank accession nos EU395595EU395601) were also analyzed as additional reference sequences [44]. Aligned sequences were exported as FASTA alignment for statistical analysis using the DnaSP v5.10.01 software [45]. Sequencing was repeated to confirm singletons. The numbers of segregating sites $(S)$, the total number of mutations $(\eta)$, the average number of pairwise nucleotide differences $(k)$, nucleotide diversity $(\pi)$, the number of haplotypes $(H)$ and haplotype diversity $(H d)$, and their corresponding standard deviation were computed using the options available in DnaSP v5.10.01 software [45]. The distribution of nucleotide diversity $(\pi)$ across the DI-III of Pvamal gene was analyzed using the sliding window approach.

\section{Haplotype network construction, linkage disequilibrium (LD) and $F_{S T}$ analysis}

A haplotype network based on the Pvama1 sequence was constructed by using the NETWORK software Version 4.6.1.3 with the Median-Joining method [46]. A total of 534 publically available Pvama1 sequences representing seven $P$. vivax populations were retrieved from the GenBank: South Korea [47], India [38], Sri Lanka [32], Thailand [48], Iran [49], Venezuela [50], and Papua New Guinea (PNG) [37]. LD was performed to calculate the minimum number of recombination events $\left(R_{\mathrm{M}}\right)$ [51] and to give an estimation of the recombination parameter $C$ [52]. The relationship between LD and distance between nucleotide sites was plotted by using indices $D^{\prime}$ [53] and $R^{2}$ [54]. To assess the proportion of genetic variance due to population subdivision, Wright's $F_{S T}$ among populations were calculated by using DnaSP v5.10.01 [45].

\section{Statistical and phylogenetic analysis}

To determine departure from neutrality, Tajima's $D, \mathrm{Fu}$ and Li's $D^{*}$ and $F^{*}$, and McDonald-Kreitman indices were calculated using DnaSP v5.10.01 and via a sliding window method [45]. In Tajima's $D$ test, departure from neutrality is determined by comparing the values of $\theta$ (estimated nucleotide diversity) derived from $\pi$ (observed average pairwise nucleotide diversity) and the total number of segregating sites $(S)$ [55]. The Fu and Li's $D^{*}$ and $F^{*}$ tests determine departure from neutrality by differences between estimation of $\theta$ derived from the number of singletons and that based on either the total number of mutations $\left(D^{*}\right)$ or the average pairwise diversity $\left(F^{*}\right)$ [56]. The McDonald-Kreitman test [57] was applied by using a single $P$. cynomolgi ama1 sequence (GenBank accession no. X86099; [58]) as the outgroup for comparison with the $P$. vivax populations. It allows for determination of the ratio of synonymous substitutions to nonsynonymous substitutions between and within species. A two-tailed Fisher's exact test was computed to determine the statistical significance $(P<0.05)$. A phylogenetic analysis of Pvama1 sequences (nt 322-737 relative to Sal-I strain) with Myanmar samples [59] was performed by the neighborjoining (NJ) method in the MEGA 6.0 software [43]. The bootstrap method with 1,000 replications was used to assess the reliability of the gene tree.

\section{Structural modelling of PvAMA1}

The potential B cell epitopes of DI-III in PvAMA1 were predicted by using the ABCpred server [60]. In order to consider a given region as a valid linear B-cell epitope, a threshold of 0.7 was used to predict the 16 residues. The prediction of intrinsically unstructured/disordered regions (IURs) in proteins, which is highly desirable in the design of vaccines and diagnostic tests, was carried out using the RONN server [61]. Residues with an output score above 0.5 were regarded as disordered. The overlapped regions between B-cell epitopes and IURs, as well as mutation sites across DI-III were mapped on a three dimensional structure of PvAMA1 (Protein Data Bank ID: 1W8K; [28]). ViewerLite 4.2 was used to visualize the surface distribution of mapped IURs, the polymorphism, and B-cell epitopes on the crystal structure of PvAMA1.

\section{Results}

Genetic diversity of Pvama1 among $P$. vivax isolates from the China-Myanmar border

The 1,290 bp sequence corresponding to the ectodomain (nt 148-1,437 encoding amino acids 50-479 relative to the Sal-I strain) of Pvama1 was amplified from 73 P. vivax samples acquired from the China-Myanmar border. Of 
the 73 Pvama1 sequences, there were 41 haplotypes, giving overall haplotype diversity $(H d)$ of 0.958 (Table 1$)$. The average number of pairwise nucleotide differences $(k)$ for the entire 1,290 bp sequenced region, DI, DII, and DIII were $10.895,7.433,1.385$, and 0.419 , respectively (Table 1 ). A total of 46 SNPs was detected, including 13 synonymous and 33 non-synonymous (Table 2). Nucleotide diversity $(\pi)$ at DI, DII and DIII of Pvama1 among 73 examined $P$. vivax isolates was $0.016,0.005$, and 0.002 , respectively, giving an overall $\pi$ value of the sequenced region of 0.008 (Table 1). A sliding window plot of $\pi$ with a window of $90 \mathrm{bp}$ and step size of $3 \mathrm{bp}$ revealed values ranging from 0.000 to 0.023 (Fig. 1a). The ectodomain contained 43 polymorphic sites, of which four (nt 162, 957, 1377, 1412) were novel compared to previous reports [32, 37, 38, 47-50]. The majority of polymorphic sites were identified in DI (26 sites) and DII (5 sites) as compared to two sites in DIII (Fig. 1b). Of these polymorphic sites, five were trimorphic, while the rest were dimorphic, displaying only two alternative amino acids (Fig. 1b). Within DI, 10 of the 26 Pvama 1 mutations were mapped to the same positions as the Pfama1 c1, c1L, c2, and c3 clusters. Among them, four mapped to the $\mathrm{c} 1$ cluster, and one was located within the Pvama1 c1L cluster (Additional file 2: Figure S1).

\section{Genetic diversity of Pvama1 in the eight worldwide parasite populations}

The Pvama1 sequences obtained from the China-Myanmar border $(n=73)$ were then compared to previously published sequences from seven other geographic regions and primate-adapted $P$. vivax isolates (nt 148-1,437 encoding amino acids 50-479). A total of 117 mutations were identified among the 615 global and reference Pvama1 sequences, including 38 synonymous SNPs (SP SNPs) and 79 non-synonymous SNPs (NS SNPs) (Table 2). The majority of the NS SNPs (53.2\%) were clustered within DI, resulting in a peak nucleotide diversity $(\pi=0.020)$ for this region (Additional file 3: Figure S2). A total of 308 haplotypes were identified among these global isolates, demonstrating an extremely high level of haplotype diversity $(H d=0.988)$. Nucleotide diversity was the highest in the Iranian and Thai populations and lowest in the South Korean population (Table 2). Despite that the majority of haplotypes was unique to the China-Myanmar border and Myanmar populations, Pvamal sequences from the China-Myanmar border population did not form distinct clades with the Myanmar isolates [59] by phylogenetic analyses (Additional file 4: Figure S3).

\section{Recombination and linkage disequilibrium}

Analysis of the ectodomain of Pvama1 from the ChinaMyanmar border samples provided estimates of the minimum number of recombination events of six, while values of the recombination parameter $C$ between adjacent sites and per gene were 0.043 and 25.5 , respectively (Table 3). These values within the China-Myanmar border population were higher compared to estimates from the South Korea, India, Sri Lanka and Venezuela populations, but lower than those of the Thailand, PNG and Iran populations, respectively (Table 3 ). Figure 2 shows the relationship between $R^{2}, D^{\prime}$ with distance between sites. This LD analysis showed a decline of the LD index $\left(R^{2}\right)$ with increasing nucleotide sites distance within the Pvama1 gene of the China-Myanmar border isolates, indicating a high meiotic recombination rate (Fig. 2).

\section{$F_{S T}$ analysis}

In order to understand the distribution of diversity across global populations, $F_{S T}$ values of the China-Myanmar border population and seven worldwide populations with full-length ectodomain sequences were evaluated [45]. A high level of genetic differentiation $\left(F_{S T}=0.47\right)$ was detected between the South Korean population and the China-Myanmar border population, whereas a low to moderate level of genetic differentiation was detected among other worldwide populations (ranging from 0.03 to 0.24$)$. A moderate range of $F_{S T}$ values $(0.13-0.16)$ were detected when comparing the China-Myanmar border populations with the India, Iran, Venezuela, and PNG populations, but a much lower genetic difference

Table 1 Nucleotide diversity and summary statistics of Pvama1 in 73 P. vivax isolates from the China-Myanmar border area. The total sequenced region includes codons 50 to 479: Domain I codons 94 to 247 (nt, 280-741), Domain II codons 265 to 363 (nt, 793-1,089), and Domain III codons 388 to 451 (nt, 1,162-1,353)

\begin{tabular}{llllllllllll}
\hline & $k$ & $H$ & $H d \pm S D$ & $S$ & $\eta$ & $\pi \pm S D$ & $\theta \pm S D$ & $D$ & $D^{*}$ & $F^{*}$ & $M K$ \\
\hline Total & 10.895 & 41 & $0.958 \pm 0.014$ & 43 & 46 & $0.008 \pm 0.000$ & $0.007 \pm 0.002$ & 0.495 & 0.772 & 0.796 & $0.0001^{* * *}$ \\
D I & 7.433 & 27 & $0.919 \pm 0.022$ & 26 & 28 & $0.016 \pm 0.000$ & $0.012 \pm 0.004$ & 0.912 & 0.269 & 0.608 & $0.004^{* *}$ \\
D II & 1.385 & 9 & $0.783 \pm 0.031$ & 5 & 5 & $0.005 \pm 0.000$ & $0.003 \pm 0.002$ & 0.780 & 1.064 & 1.142 & 0.064 \\
D III & 0.419 & 3 & $0.330 \pm 0.004$ & 2 & 2 & $0.002 \pm 0.000$ & $0.002 \pm 0.002$ & 0.031 & 0.712 & 0.589 & 0.478
\end{tabular}

Abbreviations: $S D$ standard deviation, $k$ the average number of nucleotide differences, $H$ number of haplotypes, $H d$ haplotype diversity, $S$ number of polymorphic (segregating) sites, $\eta$ the total number of mutations, $\pi$ pairwise nucleotide diversity, $\theta$ the expected nucleotide diversity under neutrality derived from $S$. $D$ Tajima's $D$ test, $D^{*} \mathrm{Fu}$ and Li's $D^{*}$ value, $F^{*}$ Fu and Li's $F^{*}$ value, MK McDonald-Kreitman test

${ }^{* *} P<0.01 ;{ }^{* * *} P<0.0001$ 
Table 2 Genetic diversity of Pvama1 in eight worldwide populations

\begin{tabular}{llllllllllll}
\hline Region & Country & $n$ & $S$ & $\eta$ & $k$ & $\pi \pm S D$ & $N S$ & $S P$ & $H$ & $H d \pm S D$ & GenBank accession numbers \\
\hline East Asia & China-Myanmar & 73 & 43 & 46 & 10.895 & $0.008 \pm 0.000$ & 33 & 13 & 41 & $0.958 \pm 0.014$ & KX495505-KX495577 \\
& Korea & 66 & 56 & 56 & 7.620 & $0.006 \pm 0.000$ & 35 & 21 & 27 & $0.950 \pm 0.013$ & KM230319-KM230384 \\
South Asia & India & 10 & 27 & 25 & 10.822 & $0.008 \pm 0.001$ & 20 & 5 & 7 & $0.911 \pm 0.077$ & EF025187-195, 197 \\
& Sri Lanka & 23 & 34 & 32 & 10.130 & $0.008 \pm 0.000$ & 27 & 5 & 15 & $0.949 \pm 0.028$ & EF218679-EF218701 \\
Southeast Asia & Thailand & 231 & 52 & 46 & 11.927 & $0.009 \pm 0.000$ & 34 & 12 & 94 & $0.934 \pm 0.012$ & FJ784891-FJ785121 \\
West Asia & Iran & 29 & 40 & 43 & 12.936 & $0.010 \pm 0.000$ & 34 & 9 & 29 & $1.000 \pm 0.009$ & JX624732-JX624760 \\
South America & Venezuela & 73 & 27 & 25 & 9.419 & $0.007 \pm 0.000$ & 19 & 6 & 18 & $0.909 \pm 0.016$ & EU346015-EU346087 \\
Oceania & PNG & 102 & 39 & 38 & 10.668 & $0.008 \pm 0.000$ & 31 & 7 & 83 & $0.995 \pm 0.002$ & KC702402-KC702503 \\
& Reference strains & 8 & 43 & 45 & 15.607 & $0.012 \pm 0.002$ & 36 & 9 & 7 & $0.964 \pm 0.077$ & EU3955595-EU395601 \\
Total & & 615 & 130 & 117 & 13.497 & $0.010 \pm 0.000$ & 79 & 38 & 308 & $0.988 \pm 0.002$ &
\end{tabular}

Abbreviations: $n$ number of samples, $S$ number of polymorphic sites, $\eta$ the total number of mutations, $k$ the average number of nucleotide differences, $\pi$ nucleotide diversity, NS number of non-synonymous polymorphisms, SP number of synonymous polymorphisms, $H$ number of haplotypes, $H d$ haplotype diversity, SD standard deviation

was identified when comparing the China-Myanmar border population with the Thai population $\left(F_{S T}=0.03\right)$ (Table 4). Low $F_{S T}$ values $(0.01-0.10)$ were also observed when comparing part of Pvama1 DI (nt 322-737) among Myanmar, Thai and China-Myanmar border populations (Additional file 5: Table S2).

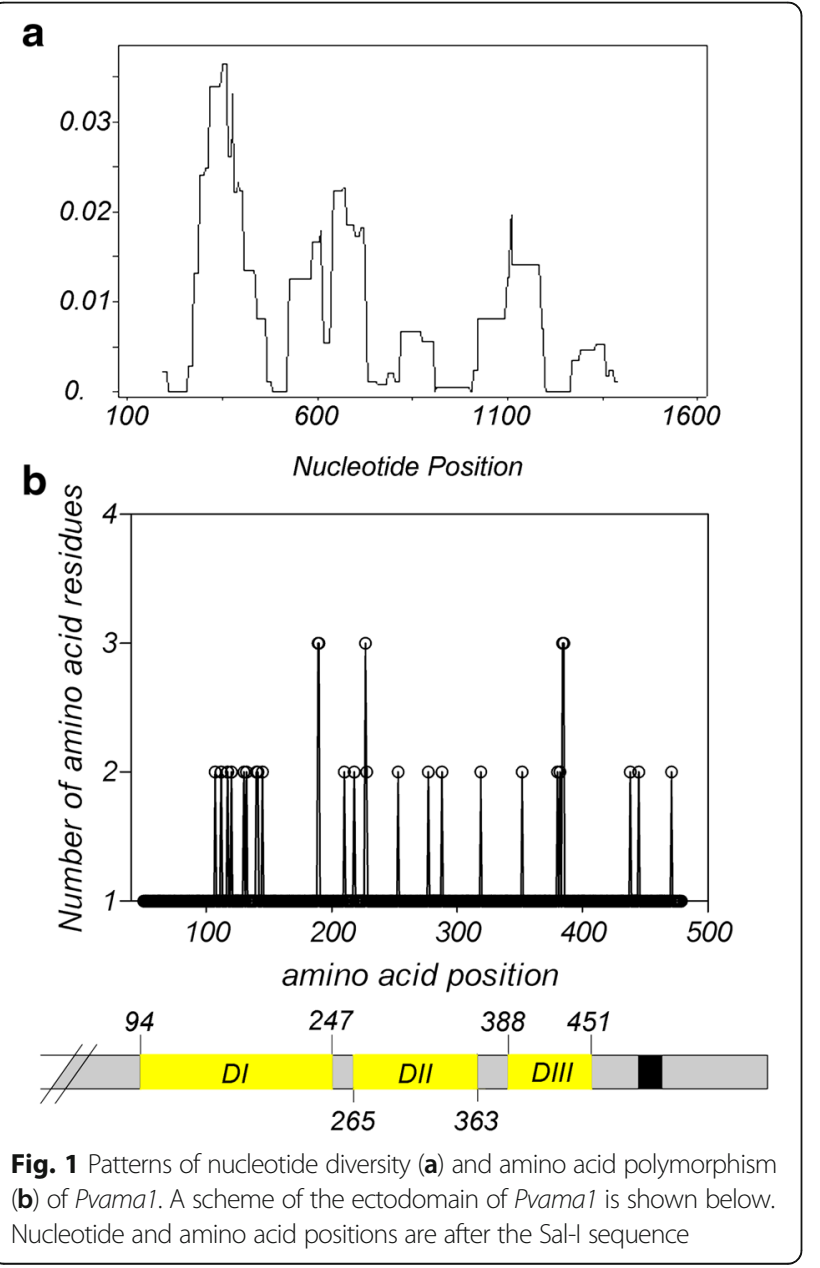

Evidence of positive diversifying selection on Pvama1 gene To examine whether natural selection contributed to the generation of the diversity in Pvama1 of the ChinaMyanmar border $P$. vivax population, Tajima's $D$ and $\mathrm{Fu}$ and Li's $D^{*}$ and $F^{*}$ were performed. The values of Tajima's $D$ and Fu and Li's $D^{*}$ and $F^{*}$ indices for the entire ectodomain and each domain separately are displayed in Table 1. Even though these values were not statistically significant, the positive value indicates deviation from neutral evolution and the tendency for positive diversifying selection. Meanwhile, a sliding window plot depicted significant positive values in DI, suggesting positive diversifying selection in this region (Fig. 3). Examination of the two sequence sets using a McDonald-Kreitman test showed that the entire sequenced region, particularly within DI, had significantly more non-synonymous substitutions than expected from comparison with $P$. cynomolgi $(P<0.0001$ and $P=0.0037)$. This result suggests that polymorphisms found for Pvama1, especially in the DI, are maintained by diversifying selection, presumably due to host immune pressure.

Table 3 Comparison of different estimates of recombination events in Pvamal among worldwide isolates

\begin{tabular}{llll}
\hline Locality (no.) & $\mathrm{R}^{\mathrm{a}}$ & $\mathrm{R}^{\mathrm{b}}$ & $\mathrm{Rm}$ \\
\hline China-Myanmar (73) & 0.043 & 25.5 & 6 \\
South Korea (66) & 0.003 & 1.6 & 2 \\
India (10) & 0.036 & 21.1 & 5 \\
Sri Lanka (23) & 0.012 & 7.2 & 5 \\
Thailand (231) & 0.047 & 27.8 & 10 \\
Iran (29) & 0.067 & 39.9 & 8 \\
Venezuela (73) & 0.021 & 12.5 & 5 \\
PNG (102) & 0.054 & 32 & 8
\end{tabular}

Abbreviations: $R^{a}$ recombinant parameter between adjacent sites, $R^{b}$ recombinant parameter for the whole gene, $R m$ minimum number of recombination events, China-Myanmar China-Myanmar border population, PNG Papua New Guinea population 

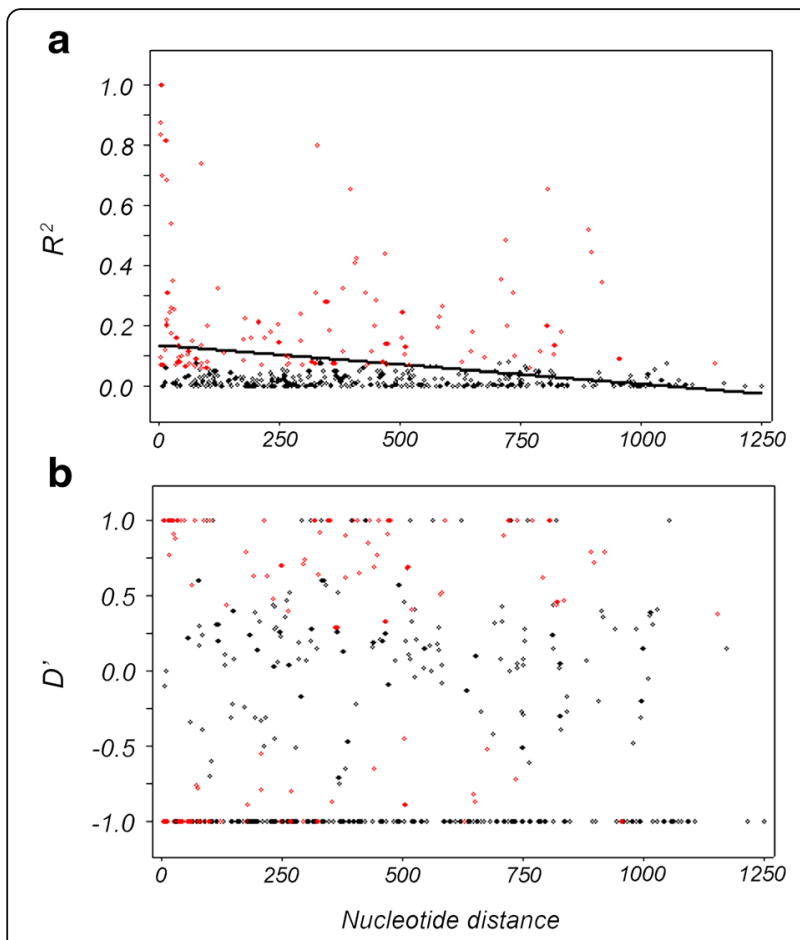

Fig. 2 Linkage disequilibrium plots of $R^{2}$ (a) and $D^{\prime}$ (b) for Pvama1 gene. Sites with significant linkage $(P<0.05)$ as calculated by Fisher's exact test are shown as red circles, while all others are shown as black circles. Trace line represents the regression line

\section{Haplotype network reconstruction}

On the basis of the 34 polymorphisms in the Pvama1 DI global isolates, 162 unique haplotypes were identified in 615 sequences, of which $54.9 \%$ was singleton. Individual haplotype prevalence ranged from 0.6 to $45.1 \%$. Of the reference strain Pvama1 sequences, only the Belem haplotype was detected, but was restricted to the Thai population. No haplotype matched that of the Sal-I strain. Twelve of the 27 China-Myanmar border haplotypes $(44.4 \%)$ were shared with other populations, of which $91.7 \%$ (11/12) were identical to some of the Pvama1 haplotypes observed in the Thai population.
Haplotype 21 is the predominant haplotype both in the China-Myanmar border and Thai populations, with a frequency of $24.7 \%$ and $23.8 \%$, respectively. Intriguingly, an independent haplotype analysis performed among the China-Myanmar border, Myanmar, and Thai populations indicates that Haplotype 21 is also the only haplotype shared among these three populations (Additional file 6: Figure S4). Haplotype 10 is shared among the ChinaMyanmar border, Indian, Sri Lankan, Thai and Iranian populations, with a frequency of $2.7,10.0,30.4,2.6$, and $10.3 \%$, respectively. Haplotype 30 is the only haplotype shared between the China-Myanmar border population and the outside Asian population. The haplotype network, drawn by excluding the 89 singletons and $\leq 2 \%$ frequency haplotypes from the analysis, showed that clusters from the South American, Oceania, and Asian populations overlapped (Fig. 4).

\section{Analysis of PvAMA1 structure and antigenicity}

To determine the distribution of the B-cell epitopes, IURs, and the polymorphic residues found within the ChinaMyanmar border population, the modeled structure of PvAMA1 was used. The potential B-cell epitopes were identified across the ectodomain of PvAMA1, which also overlap with the locations of most of the SNPs (Additional file 7: Figure S5). Also, the putative IURs are distributed in five regions (amino acids - aa: 121-141, 171-183, 291309, 392-406, and 427-441) within all three domains of the PvAMA1 protein with no polymorphic residues detected in aa 171-183 and 291-309. In Fig. 5, the overlap between the putative B-cell epitopes and IURs have been demonstrated in the 3-D model of PvAMA1 in yellow (with polymorphic residues, aa: 121-141, 392-406, and 427-441) and red colors (without polymorphic residues, aa: 171-183 and 291-309), respectively. The polymorphic residues clustered on one side of the PvAMA1 protein. Interestingly, the two overlapping non-polymorphic residues of the B-cell epitopes and IURs are located on the opposing faces of the PvAMA1 protein (Fig. 5).

Table 4 Geographical difference in Pvamal sequences across all domains

\begin{tabular}{|c|c|c|c|c|c|c|c|}
\hline Locality $(n)$ & China-Myanmar & South Korea & India & Sri Lanka & Thailand & Iran & Venezuela \\
\hline \multicolumn{8}{|c|}{ China-Myanmar (73) } \\
\hline South Korea (66) & 0.47 & & & & & & \\
\hline India (10) & 0.13 & 0.39 & & & & & \\
\hline Sri Lanka (114) & 0.24 & 0.54 & 0.20 & & & & \\
\hline Thailand (231) & 0.03 & 0.47 & 0.12 & 0.21 & & & \\
\hline Iran (29) & 0.11 & 0.37 & 0.07 & 0.10 & 0.12 & & \\
\hline Venezuela (73) & 0.12 & 0.48 & 0.17 & 0.25 & 0.17 & 0.01 & \\
\hline PNG (102) & 0.19 & 0.42 & 0.06 & 0.22 & 0.17 & 0.26 & 0.25 \\
\hline
\end{tabular}

Abbreviations: China-Myanmar China-Myanmar border population, PNG Papua New Guinea population 

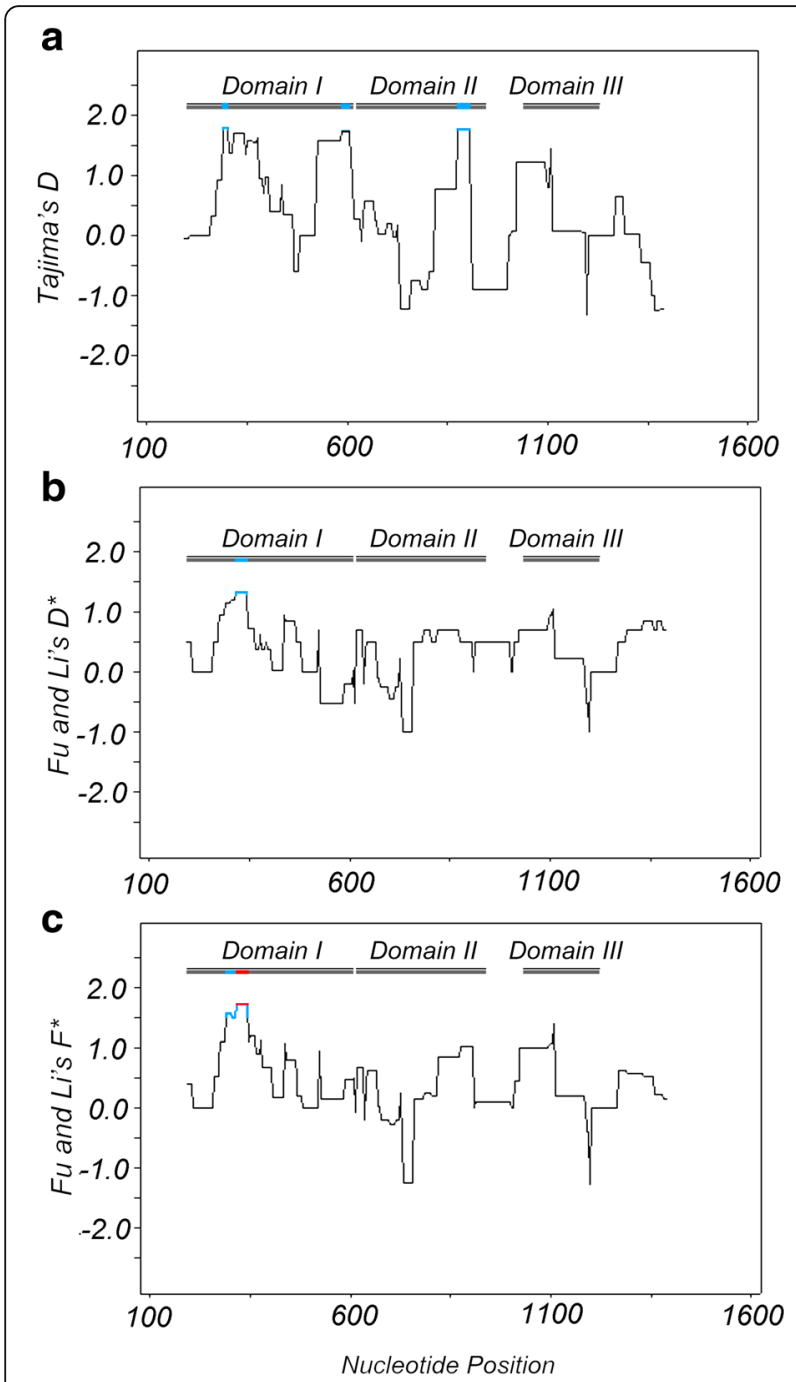

Fig. 3 Natural selection on Pvama1 isolates from the China-Myanmar border area. Sliding-window plots of Tajima's D test (a), Fu and Li's D* (b) and $F^{*}$ (c) tests for Pvamal were shown. Nucleotide numbers follow those of Sal-I. Window length is $90 \mathrm{bp}$, and step size is $3 \mathrm{bp}$. The three domains of AMA1 (Domain I, Domain II, and Domain III) are shown. Blue lines indicate the region with a significant departure from neutrality $(P<0.1$, one-tailed). Red lines indicate regions with positive values $(P<0.05$, one-tailed $)$

\section{Discussion}

Consistent with worldwide reports, we detected high genetic diversity in the ectodomain of Pvama1 sequences obtained in 73 P. vivax isolates from the China-Myanmar border. Globally, substantial geographic variability has been observed, with lower diversity in East Asian (Korean, $\pi=0.006$ [47]) and South American (Venezuelan, $\pi=$ 0.007 [50]) populations, compared to higher diversity in Southeast Asian (Thai, $\pi=0.009$ [48]) and West Asian (Iranian, $\pi=0.010$ [49]) populations. As genetic diversity of $P$. vivax populations varies significantly due to transmission intensity [62-64], it is likely that the higher genetic diversity observed in Thailand and Iran was due to higher $P$. vivax transmission intensity than in Korea and Venezuela. The detected high level of genetic diversity in Pvama1 in parasite population from the China-Myanmar border was not in line with the rapid decline of malaria incidence in recent years. While it is expected that genetic diversity diminishes following the reduction in malaria incidence, the relationship between the two aspects is complex and could vary according to many epidemiological parameters. In Thailand, for example, $P$. vivax population in the western border was highly diverse as compared to being almost clonal in the southern border $[9,10]$. It remains to be determined whether these differences were due to different histories of malaria control efforts and levels of parasite introduction from neighboring highly endemic areas. Nevertheless, with the overall increased control efforts in the GMS and more isolated parasite populations on the international borders, future longitudinal tracking of parasite genetic diversity in the local parasite populations as well as in potential sources of parasite introduction will provide answers to these questions. Meanwhile, the use of more than one genetic marker such as a molecular barcode may provide a better resolution of the parasite populations [65].

The LD indices decline rapidly with the increasing distance between sites. Considering the high values of recombination parameter, $C(C=4 N r)$, these results indicate that recombination is very frequent in the Pvamal gene of diverse geographic populations [32, 37, 38, 47-50]. An estimated moderate level of the recombination parameter $C$ (both between adjacent site, $\mathrm{R}^{\mathrm{a}}$ and per gene, $\mathrm{R}^{\mathrm{b}}$ ) was detected for Pvama1 (0.043 and 25.5) in the ChinaMyanmar border population. This $C$ value observed for Pvama1 is much higher than that of Pfama1 (0.003 and 3.8) from a sympatric population of $P$. falciparum [66], for which the effective population size $\left(N_{e}\right)$ is only $1.3 \times 10^{3}$ when calculated by using the average recombination rate of $P$. falciparum $\left(\sim 6 \times 10^{-7}\right.$ Morgans/base) [67]. We conclude from these data that recombination events in Pvama1 of the China-Myanmar border population are common, and that this area appears to have a considerably larger effective population of $P$. vivax than $P$. falciparum. In $P$. falciparum, strong LD and low genetic diversity often are observed in low-transmission areas [68]. However, such a spectrum of population structures may not fit for $P$. vivax populations, as we observed LD and high-level haplotype diversity (0.958) of Pvama1 in the China-Myanmar border, where malaria transmission is low [8]. We speculate that two factors might contribute to the observed high genetic diversity in Pvamal in the border regions. The ability to relapse in $P$. vivax increases the prevalence of mixed infections and chances for genetic 


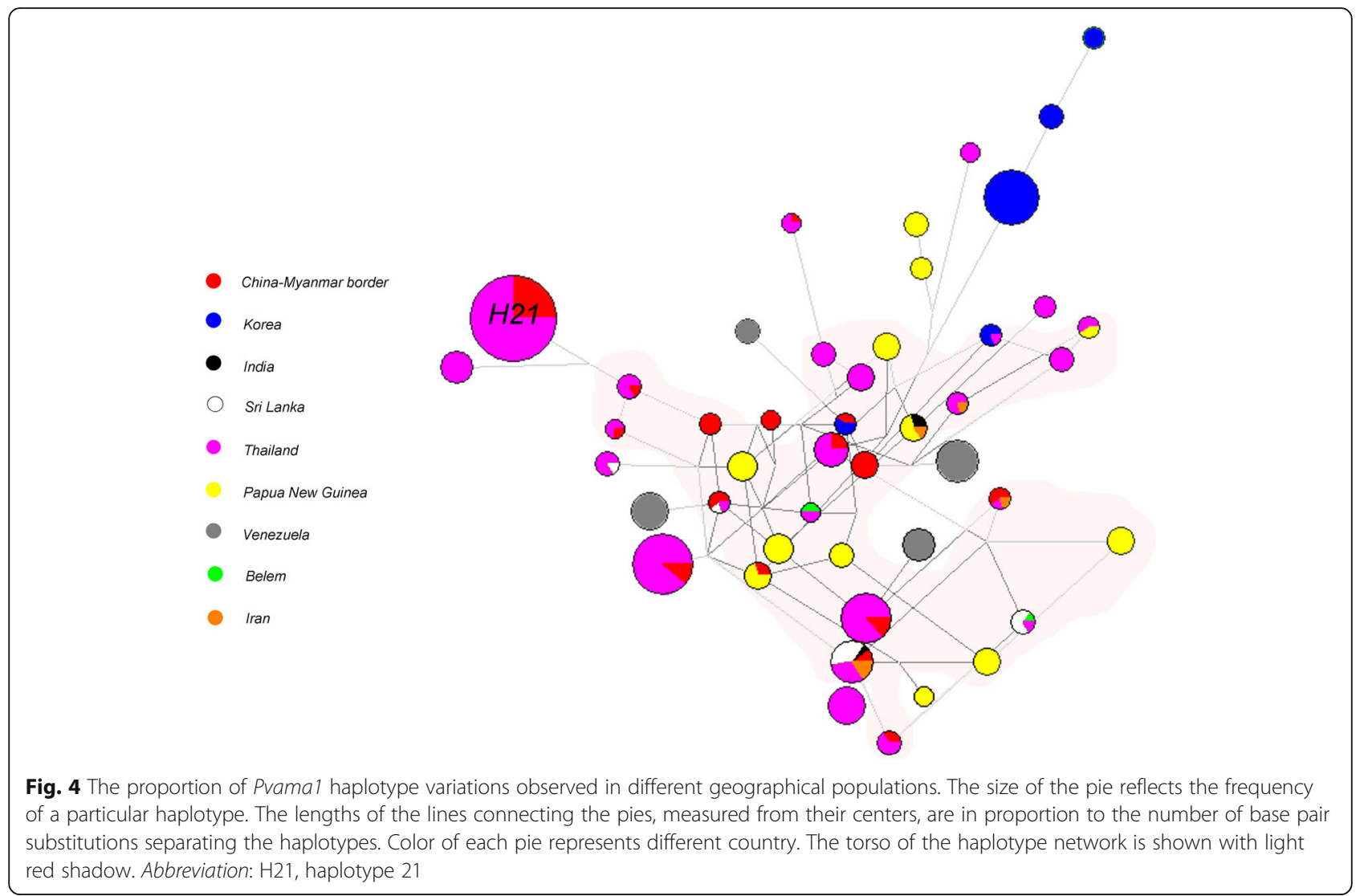

recombination in the mosquito vectors. In addition, heavy human population flow across the porous international borders further favors mixing of parasite populations and increasing parasite genetic diversity.

Pairwise $F_{S T}$ comparisons only detected a moderate level of genetic differentiation $\left(F_{S T}=0.23\right)$ among global populations. This may reflect the functional constraints of this protein and similar balancing selection from human immunity on this protein. Despite this, major geographical genetic differentiation does exist. For instance, the Korean parasite population was drastically different from other parasite populations from the world. The
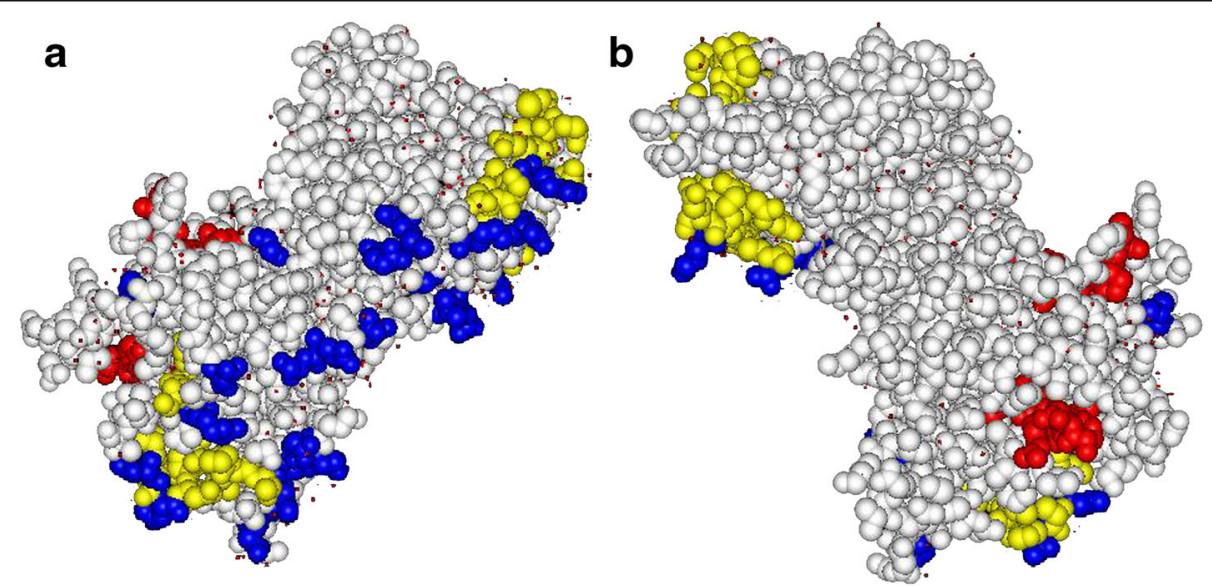

Fig. 5 CPK-models of PVAMA1 with IURs, predicted B-cell epitopes, and polymorphic residues across DI-III mapped. Two opposite sides of the same 3-D structure are shown in (a) with and in (b) without the polymorphic residues. Residues in overlapped regions of B-cell epitopes and IURs with polymorphic residues (aa 121-141, 392-406, and 427-441) are shown in yellow, while overlapped regions without polymorphic residues (aa 171-183 and 291-309) are shown in red. Polymorphic residues across DI-III are shown in pink. Other amino acids are shown in white 
Thai $P$. vivax populations collected from three regions (northwestern, eastern and southern) from 1996 and 2007 also showed significant population subdivision [48], which may be due to temporal and spatial separations among these populations. Later studies confirmed that the southern Thai population had experienced a genetic bottleneck with significantly reduced genetic diversity $[9,10]$. Yet, very little genetic differentiation was observed between the combined Thai parasite population and the China-Myanmar border population, despite that our parasite samples were acquired more recently. This demonstrates the persistence of highly diverse $P$. vivax populations with only slight genetic differentiation in different regions of the GMS, suggesting the lack of major gene flow barriers in this region. Together with the fact that similar allelic forms of Pvama1 are circulating in the Myanmar, China-Myanmar border, and Thai $P$. vivax populations $[48,59]$, the low genetic differentiation between these regions support the idea that a general malaria elimination or control strategy may be applicable for these endemic regions.

The pattern of polymorphisms observed in the ectodomain as well as DI of the China-Myanmar border Pvama1 showed that both domains are under diversifying selection, suggesting a significant departure from neutrality as confirmed by the McDonald and Kreitman test. These results are consistent with previous reports of Pvama1 DI from various global isolates [32, 37, 38, 47-50]. Although DII of Pvamal has been reported as highly immunogenic $[33,69]$, and there is evidence of positive selection in DII of Sri Lankan P. vivax populations [32], no evidence was found for diversifying selection on DII and DIII of the China-Myanmar border Pvama1 as confirmed by neutrality tests, which is in agreement with several other reports $[37,50]$. These results suggest that DI is the dominant target of the host immune response.

A total of 308 haplotypes, with a haplotype diversity of 0.988 , was identified in global isolates when analyzing the ectodomain of Pvama1. This extreme polymorphism indicates that each haplotype might be recognized as immunologically distinct by the immune system. Further observation of Pvama1 DI showed that only haplotype 30 in the China-Myanmar border is shared with populations outside of Asia, and haplotype 10 is the only one shared among the majority of analyzed Asian populations. Together with the complex network analysis results, covering diversity of $P$. vivax will be a great challenge for a PvAMA1-based vaccine design. Although highly diverse, the observation that the majority of the China-Myanmar border Pvama 1 haplotypes (44.4\%) are shared with Thai populations and that the dominant haplotype $(\mathrm{H} 21)$ is identical in both populations suggested little differentiation of parasite populations within GMS, and a PvAMA1-based malaria vaccine may be effective in this entire region. Notably, the vaccine strain Sal-I haplotype was not identified in any of the analyzed population in the current study. Thus, the dominant Pvama1 alleles from field parasite populations should be considered in the future studies.

Compared to the Sal-I sequence, 46 SNPs resulting in 43 amino acid substitutions were identified in the ChinaMyanmar border Pvama1. The majority of the diversity clustered within Pvama1 DI, as has been reported previously for P. falciparum and P. vivax [29, 37, 50]. The 90.7\% (39/43) polymorphic sites within the ectodomain identified in the China-Myanmar border Pvama1 are comparable to those previously reported [32, 37, 38, 47-50], indicating that these sites may be under strong natural selection. Four clusters within DI (c1, c1L, c2 and c3) were mapped to the surface of the PfAMA1 protein and are potentially associated with antigenic escape [70, 71]. In P. falciparum, the $\mathrm{c} 1$ (positions 187 to 231) and c1L (positions 196-207) clusters contain the most polymorphic residues [70, 71]. However, only four Pvama1 polymorphic sites of the ChinaMyanmar border isolates were located in the Pfama1 c1 cluster. These mutation sites are comparable with those observed in the PNG isolates [37], supporting the hypothesis that the presentation of the PvAMAl's DI loops is different from that of PfAMA1. Structural modeling of PvAMA1 from the China-Myanmar border isolates revealed that all of the polymorphic residues are mapped to one surface of the PvAMA1 protein, suggesting this is the side face exposed to the host immune system [29]. Previous studies by using invasion-inhibitory monoclonal antibodies specific for PfAMA1 revealed that DII of PvAMA1 is a target of the protective immune response. Furthermore, IUR region (aa 290-307) in the DII of PvAMA1 was found to be an important antigenic region during natural human infections [52]. Comparable to these previous reports, amino acid sequence conservation has also been identified in China-Myanmar border isolates, with no polymorphic sites in aa 171-183 (in DI) and 291-309 (in DII) of B-cell epitope and IUR overlapped region have been identified in all 73 sequenced samples. The current results support the inclusion of amino acid sequences aa 291-309 in DII in PvAMA1based vaccine.

\section{Conclusions}

Our study demonstrated that the genetic diversity of the Pvama1 in 73 P. vivax isolates from the China-Myanmar border area, as well as global isolates, was exceptionally high, and that Pvama1 DI is the dominant target of positive diversifying selection. Furthermore, a low level of genetic differentiation among the Myanmar, China-Myanmar border and Thai populations suggests high levels of gene flow within the GMS. 


\section{Additional files}

Additional file 1: Table S1. Oligonucleotides used for amplification and sequencing of Pvamal gene sequences. (DOCX $14 \mathrm{~kb}$ )

Additional file 2: Figure S1. Alignment of $P$. falciparum, $P$. vivax, and $P$. cynomolgi AMA1 protein sequences. P. falciparum 3D7 (PlasmoDB ID: PF3D7_1133400), P. vivax Sal-I (GenBank access no. AF063138) and P. cynomolgi (GenBank access no. X86099) were aligned using MUltiple Sequence Comparison by Log-Expectation (MUSCLE, http://www.ebi.ac.uk/ Tools/mas/muscle/). Gaps are indicating by dashes. Conserved amino acids among the three analyzed strains are marked by asterisks. Red bold types indicate conserved cysteine residues that divide the ectodomain of AMA1. Domains I-III is shown in grey, green and yellow color, respectively. Boxes indicate $\mathrm{c} 1, \mathrm{c} 2$, and $\mathrm{c} 3$ clusters. The $\mathrm{c} 1 \mathrm{~L}$ cluster region is marked with underlines. Light blue bold type indicates residues that are polymorphic in P. vivax of China-Myanmar border isolates. (TIF 2305 kb)

Additional file 3: Figure S2. Patterns of nucleotide diversity and amino acid polymorphisms of Pvama1. Sliding window plot of nucleotide diversity ( $\pi$ ) and amino acid polymorphism of Pvamal ectodomain in 607 global isolates and 8 reference strains were shown. The $\pi$ value was calculated using DnaSP v5.10.01 with window length of $90 \mathrm{bp}$ and step size of $3 \mathrm{bp}$ Domain I is highlighted in red color. Nucleotide and amino acid positions are after the Sal-I sequence. (TIF $183 \mathrm{~kb}$ )

Additional file 4: Figure S3. Phylogenetic analysis of China-Myanmar border and Myanmar Pvamal sequences. The tree was constructed by analyzing DI (nt, 322-737) with 73 Pvamal sequences obtained from China-Myanmar border and 24 Pvamal sequences obtained from Myanmar P. vivax isolates (GenBank accession nos. FJ157247, FJ157249, FJ157251, FJ157253-FJ157269 and FJ157282-FJ157285) using a neighbor-joining method. The bootstrap method with 1,000 replications was used to construct the gene tree. Red circle indicate sequences obtained from Myanmar. (TIF $1803 \mathrm{~kb}$ )

Additional file 5: Table S2. Genetic differentiation $\left(F_{S T}\right)$ of the Pvama1 gene among Thai, Myanmar and China-Myanmar border populations across DI. (DOCX $14 \mathrm{~kb}$ )

Additional file 6: Figure S4. The proportion of Pvama1 haplotypes variation observed in China-Myanmar border, Myanmar, and Thai populations. The size of the pies reflects the frequency of a particular haplotype. The lengths of the lines connecting the pies, measured from their centers, are in proportion to the number of base pair substitutions separating the haplotypes. Color of each pie represents different country. Abbreviation: H21, haplotype 21. (TIF $831 \mathrm{~kb}$ )

Additional file 7: Figure S5. Location of current study detected SNPS in the predicted B-cell epitopes and IURs in PVAMA1. Predictions of B-cell epitopes and IURs were performed by using ABCpred and RONN server, respectively. The cysteine residues have been shown in red bold. DI = 94-247 aa; DII = 265-363 aa; DIII = 388-451 aa; SNPs in the current study were marked in grey shadow; underlined shows B-cell epitopes; Bold overbars show IURs. (TIF $323 \mathrm{~kb}$ )

\section{Abbreviations}

C1L: Cluster 1 loop; DBP: Duffy-binding protein; DI: Domain I; DII: Domain II; DIII: Domain III; $F_{S T}$ : Fixation index; IURs: Intrinsically unstructured/disordered regions; LD: Linkage disequilibrium; MK: McDonald Kreitman test; MSP1: Merozoite surface protein 1; NS SNPs: Non-synonymous SNPS; PNG: Papua New Guinea; PvAMA1: Plasmodium vivax apical membrane antigen 1; SP SNPS: Synonymous SNPS

\section{Acknowledgements}

We would like to thank patients from the surrounding villages for participating in this study and providing the blood samples.

\section{Funding}

This work was supported by grants from NIAID, National Institutes of Health (U19AI089672) and grants from National Natural Science Foundation of China (Grant no. 81301455).

\section{Availability of data and materials}

The datasets supporting the conclusions of this article are included within the article and its additional files.

\section{Authors' contributions}

$X Z, Y C$ and LC designed the studies and drafted the manuscript. PZ and SW took part in the genomic DNA extraction, PCR amplification and writing of the manuscript. $J \mathrm{~L}, \mathrm{FL}$, and JW participated in sequence analysis and writing of the manuscript. ZY, QF and GY performed epidemiology study, sample collection and writing of the manuscript. YC and LC revised the final manuscript. All authors read and approved the final manuscript.

\section{Competing interests}

The authors declare that they have no competing interests.

\section{Consent for publication}

Not applicable.

\section{Ethics approval and consent to participate}

The study protocol was approved by the IRB of Pennsylvania State University and by the Health Department of Kachin. Written informed consent/assent was obtained from all participants.

\section{Author details}

${ }^{1}$ Department of Immunology, College of Basic Medical Science, China Medical University, Shenyang, Liaoning 110122, China. ${ }^{2}$ Department of Microbiology and Parasitology, College of Basic Medical Sciences, China Medical University, Shenyang, Liaoning 110122, China. ${ }^{3}$ Department of Pathogen Biology and Immunology, Kunming Medical University, Kunming, China. ${ }^{4}$ Program in Public Health, University of California, Irvine, CA, USA. ${ }^{5}$ Dalian Institute of Biotechnology, Dalian, Liaoning, China. ${ }^{6}$ Department of Entomology, The Pennsylvania State University, University Park, PA 16802, USA.

Received: 7 August 2016 Accepted: 21 November 2016

Published online: 29 November 2016

\section{References}

1. Gething PW, Elyazar IR, Moyes CL, Smith DL, Battle KE, Guerra CA, et al. A long neglected world malaria map: Plasmodium vivax endemicity in 2010 PLoS Negl Trop Dis. 2012;6:e1814.

2. Zhou X, Huang JL, Njuabe MT, Li SG, Chen JH, Zhou XN. A molecular survey of febrile cases in malaria-endemic areas along China-Myanmar border in Yunnan province, People's Republic of China. Parasite. 2014:21:27.

3. Hewitt S, Delacollette C, Chavez I. Malaria situation in the Greater Mekong Subregion. Southeast Asian J Trop Med Public Health. 2013:44 Suppl 1:46-72. discussion 306-307.

4. Cui L, Yan G, Sattabongkot J, Cao Y, Chen B, Chen X, et al. Malaria in the Greater Mekong Subregion: heterogeneity and complexity. Acta Trop. 2012;121:227-39.

5. Feng J, Xiao H, Xia Z, Zhang L, Xiao N. Analysis of malaria epidemiological characteristics in the People's Republic of China, 2004-2013. Am J Trop Med Hyg. 2015;93:293-9.

6. Feng J, Xiao H, Zhang L, Yan H, Feng X, Fang W, Xia Z. The Plasmodium vivax in China: decreased in local cases but increased imported cases from Southeast Asia and Africa. Sci Rep. 2015;5:8847.

7. Wang D, Li S, Cheng Z, Xiao N, Cotter C, Hwang J, et al. Transmission risk from imported Plasmodium vivax malaria in the China-Myanmar border region. Emerg Infect Dis. 2015;21:1861-4.

8. Zhou G, Sun L, Xia R, Duan Y, Xu J, Yang H, et al. Clinical malaria along the China-Myanmar border, Yunnan Province, China, January 2011-August 2012. Emerg Infect Dis. 2014;20:675-8.

9. Putaporntip C, Miao J, Kuamsab N, Sattabongkot J, Sirichaisinthop J, Jongwutiwes S, Cui L. The Plasmodium vivax merozoite surface protein 3beta sequence reveals contrasting parasite populations in southern and northwestern Thailand. PLoS Negl Trop Dis. 2014;8:e3336.

10. Jongwutiwes S, Putaporntip C, Hughes AL. Bottleneck effects on vaccinecandidate antigen diversity of malaria parasites in Thailand. Vaccine. 2010;28:3112-7

11. Rich SM, Ferreira MU, Ayala FJ. The origin of antigenic diversity in Plasmodium falciparum. Parasitol Today. 2000;16:390-6. 
12. Tsuboi T, Kappe SH, Al-Yaman F, Prickett MD, Alpers M, Adams JH. Natural variation within the principal adhesion domain of the Plasmodium vivax duffy binding protein. Infect Immun. 1994;62:5581-6.

13. de Sousa TN, Kano FS, de Brito CF, Carvalho LH. The Duffy binding protein as a key target for a Plasmodium vivax vaccine: lessons from the Brazilian Amazon. Mem Inst Oswaldo Cruz. 2014;109:608-17.

14. Versiani FG, Almeida ME, Mariuba LA, Orlandi PP, Nogueira PA. N-terminal Plasmodium vivax merozoite surface protein-1, a potential subunit for malaria vivax vaccine. Clin Dev Immunol. 2013;2013:965841.

15. Remarque EJ, Faber BW, Kocken CH, Thomas AW. Apical membrane antigen 1: a malaria vaccine candidate in review. Trends Parasitol. 2008;24:74-84.

16. Mitchell GH, Thomas AW, Margos G, Dluzewski AR, Bannister LH. Apical membrane antigen 1, a major malaria vaccine candidate, mediates the close attachment of invasive merozoites to host red blood cells. Infect Immun. 2004:72:154-8.

17. Dutta S, Haynes JD, Moch JK, Barbosa A, Lanar DE. Invasion-inhibitory antibodies inhibit proteolytic processing of apical membrane antigen 1 of Plasmodium falciparum merozoites. Proc Natl Acad Sci U S A. 2003;100:12295-300.

18. Preiser $P$, Kaviratne M, Khan S, Bannister L, Jarra W. The apical organelles of malaria merozoites: host cell selection, invasion, host immunity and immune evasion. Microbes Infect. 2000;2:1461-77.

19. Gaur D, Mayer DC, Miller LH. Parasite ligand-host receptor interactions during invasion of erythrocytes by Plasmodium merozoites. Int J Parasitol. 2004;34:1413-29.

20. Richard D, MacRaild CA, Riglar DT, Chan JA, Foley M, Baum J, et al. Interaction between Plasmodium falciparum apical membrane antigen 1 and the rhoptry neck protein complex defines a key step in the erythrocyte invasion process of malaria parasites. J Biol Chem. 2010;285:14815-22.

21. Lamarque M, Besteiro S, Papoin J, Roques M, Vulliez-Le Normand B, Morlon-Guyot J, et al. The RON2-AMA1 interaction is a critical step in moving junction-dependent invasion by apicomplexan parasites. PLoS Pathog. 2011;7:e1001276.

22. Galinski MR, Barnwell JW. Plasmodium vivax: merozoites, invasion of reticulocytes and considerations for malaria vaccine development. Parasitol Today. 1996;12:20-9.

23. Narum DL, Thomas AW. Differential localization of full-length and processed forms of PF83/AMA-1 an apical membrane antigen of Plasmodium falciparum merozoites. Mol Biochem Parasitol. 1994;67:59-68.

24. Bannister LH, Hopkins JM, Dluzewski AR, Margos G, Williams IT, Blackman MJ, et al. Plasmodium falciparum apical membrane antigen 1 (PfAMA-1) is translocated within micronemes along subpellicular microtubules during merozoite development. J Cell Sci. 2003;116:3825-34.

25. Kusi KA, Faber BW, Thomas AW, Remarque EJ. Humoral immune response to mixed PfAMA1 alleles; multivalent PfAMA1 vaccines induce broad specificity. PLoS One. 2009;4:e8110.

26. Stowers AW, Kennedy MC, Keegan BP, Saul A, Long CA, Miller LH. Vaccination of monkeys with recombinant Plasmodium falciparum apical membrane antigen 1 confers protection against blood-stage malaria. Infect Immun. 2002;70:6961-7.

27. Anders RF, Crewther PE, Edwards S, Margetts M, Matthew ML, Pollock B, Pye D. Immunisation with recombinant AMA-1 protects mice against infection with Plasmodium chabaudi. Vaccine. 1998;16:240-7.

28. Pizarro JC, Vulliez-Le Normand B, Chesne-Seck ML, Collins CR, Withers-Martinez $C$, et al. Crystal structure of the malaria vaccine candidate apical membrane antigen 1. Science. 2005;308:408-11.

29. Polley SD, Conway DJ. Strong diversifying selection on domains of the Plasmodium falciparum apical membrane antigen 1 gene. Genetics. 2001;158:1505-12

30. Ouattara A, Takala-Harrison S, Thera MA, Coulibaly D, Niangaly A, Saye R, et al. Molecular basis of allele-specific efficacy of a blood-stage malaria vaccine: vaccine development implications. J Infect Dis. 2013;207:511-9.

31. Dias S, Somarathna M, Manamperi A, Escalante AA, Gunasekera AM, Udagama PV. Evaluation of the genetic diversity of domain II of Plasmodium vivax Apical Membrane Antigen 1 (PvAMA-1) and the ensuing strain-specific immune responses in patients from Sri Lanka. Vaccine. 2011;29:7491-504.

32. Gunasekera AM, Wickramarachchi T, Neafsey DE, Ganguli I, Perera L, Premaratne $\mathrm{PH}$, et al. Genetic diversity and selection at the Plasmodium vivax apical membrane antigen-1 (PvAMA-1) locus in a Sri Lankan population. Mol Biol Evol. 2007;24:939-47.

33. Mufalo BC, Gentil F, Bargieri DY, Costa FT, Rodrigues MM, Soares IS. Plasmodium vivax apical membrane antigen-1: comparative recognition of different domains by antibodies induced during natural human infection. Microbes Infect. 2008;10:1266-73.

34. Joshi H. Markers for population genetic analysis of human plasmodia species, P. falciparum and P. vivax. J Vector Borne Dis. 2003:40:78-83.

35. Cheng Q, Saul A. Sequence analysis of the apical membrane antigen I (AMA-1) of Plasmodium vivax. Mol Biochem Parasitol. 1994;65:183-7.

36. Rodrigues MH, Rodrigues KM, Oliveira TR, Comodo AN, Rodrigues MM, Kocken $\mathrm{CH}$, et al. Antibody response of naturally infected individuals to recombinant Plasmodium vivax apical membrane antigen-1. Int J Parasitol. 2005;35:185-92.

37. Arnott A, Mueller I, Ramsland PA, Siba PM, Reeder JC, Barry AE. Global population structure of the genes encoding the malaria vaccine candidate, Plasmodium vivax apical membrane antigen 1 (PvAMA1). PLoS Negl Trop Dis. 2013;7:e2506.

38. Rajesh V, Elamaran M, Vidya S, Gowrishankar M, Kochar D, Das A. Plasmodium vivax: genetic diversity of the apical membrane antigen-1 (AMA-1) in isolates from India. Exp Parasitol. 2007;116:252-6.

39. Chung JY, Chun EH, Chun JH, Kho WG. Analysis of the Plasmodium vivax apical membrane antigen-1 gene from re-emerging Korean isolates. Parasitol Res. 2003;90:325-9.

40. Li N, Parker DM, Yang Z, Fan Q, Zhou G, Ai G, et al. Risk factors associated with slide positivity among febrile patients in a conflict zone of northeastern Myanmar along the China-Myanmar border. Malar J. 2013;12:361.

41. Zhou G, Lo E, Zhong D, Wang X, Wang Y, Malla S, et al. Impact of interventions on malaria in internally displaced persons along the China-Myanmar border: 2011-2014. Malar J. 2016;15:471.

42. Jitthai N. Migration and malaria. Southeast Asian J Trop Med Public Health. 2013;44 Suppl 1:166-200. discussion 306-167.

43. Tamura K, Stecher G, Peterson D, Filipski A, Kumar S. MEGA6: Molecular Evolutionary Genetics Analysis version 6.0. Mol Biol Evol. 2013;30:2725-9.

44. Ntumngia FB, McHenry AM, Barnwell JW, Cole-Tobian J, King CL, Adams JH. Genetic variation among Plasmodium vivax isolates adapted to non-human primates and the implication for vaccine development. Am J Trop Med Hyg. 2009;80:218-27.

45. Librado P, Rozas J. DnaSP v5: a software for comprehensive analysis of DNA polymorphism data. Bioinformatics. 2009;25:1451-2.

46. Bandelt HJ, Forster $P$, Rohl A. Median-joining networks for inferring intraspecific phylogenies. Mol Biol Evol. 1999;16:37-48.

47. Kang JM, Lee J, Cho PY, Moon SU, Ju HL, Ahn SK, et al. Population genetic structure and natural selection of apical membrane antigen-1 in Plasmodium vivax Korean isolates. Malar J. 2015;14:455.

48. Putaporntip C, Jongwutiwes S, Grynberg P, Cui L, Hughes AL. Nucleotide sequence polymorphism at the apical membrane antigen-1 locus reveals population history of Plasmodium vivax in Thailand. Infect Genet Evol. 2009:9:1295-300

49. Zakeri S, Sadeghi H, Mehrizi AA, Djadid ND. Population genetic structure and polymorphism analysis of gene encoding apical membrane antigen-1 (AMA-1) of Iranian Plasmodium vivax wild isolates. Acta Trop. 2013;126:269-79.

50. Ord RL, Tami A, Sutherland CJ. ama1 genes of sympatric Plasmodium vivax and $P$. falciparum from Venezuela differ significantly in genetic diversity and recombination frequency. PLoS One. 2008;3:e3366.

51. Hudson RR, Kaplan NL. Statistical properties of the number of recombination events in the history of a sample of DNA sequences. Genetics. 1985;111:147-64.

52. Hudson RR. Estimating the recombination parameter of a finite population model without selection. Genet Res. 1987;50:245-50.

53. Lewontin RC. The interaction of selection and linkage. I. General considerations; heterotic models. Genetics. 1964:49:49-67.

54. Hill WG, Robertson A. Linkage disequilibrium in finite populations. Theor Appl Genet. 1968;38:226-31.

55. Tajima F. Statistical method for testing the neutral mutation hypothesis by DNA polymorphism. Genetics. 1989;123:585-95.

56. Fu YX, Li WH. Statistical tests of neutrality of mutations. Genetics. 1993;133:693-709.

57. McDonald $\mathrm{JH}$, Kreitman M. Adaptive protein evolution at the Adh locus in Drosophila. Nature. 1991;351:652-4.

58. Dutta S, Malhotra P, Chauhan VS. Sequence analysis of apical membrane antigen 1 (AMA-1) of Plasmodium cynomolgi bastianelli. Mol Biochem Parasitol. 1995;73:267-70

59. Moon SU, Na BK, Kang JM, Kim JY, Cho SH, Park YK, et al. Genetic polymorphism and effect of natural selection at domain I of apical membrane antigen-1 (AMA-1) in Plasmodium vivax isolates from Myanmar. Acta Trop. 2010;114:71-5. 
60. Saha S, Raghava GP. Prediction of continuous B-cell epitopes in an antigen using recurrent neural network. Proteins. 2006;65:40-8.

61. Yang ZR, Thomson R, McNeil P, Esnouf RM. RONN: the bio-basis function neural network technique applied to the detection of natively disordered regions in proteins. Bioinformatics. 2005;21:3369-76.

62. Osier FH, Weedall GD, Verra F, Murungi L, Tetteh KK, Bull P, et al. Allelic diversity and naturally acquired allele-specific antibody responses to Plasmodium falciparum apical membrane antigen 1 in Kenya. Infect Immun. 2010;78:4625-33.

63. Karunaweera ND, Ferreira MU, Munasinghe A, Barnwell JW, Collins WE, King $\mathrm{CL}$, et al. Extensive microsatellite diversity in the human malaria parasite Plasmodium vivax. Gene. 2008;410:105-12.

64. Imwong M, Nair S, Pukrittayakamee S, Sudimack D, Williams JT, Mayxay M, et al. Contrasting genetic structure in Plasmodium vivax populations from Asia and South America. Int J Parasitol. 2007:37:1013-22.

65. Baniecki ML, Faust AL, Schaffner SF, Park DJ, Galinsky K, Daniels RF, et al. Development of a single nucleotide polymorphism barcode to genotype Plasmodium vivax infections. PLoS Negl Trop Dis. 2015;9:e0003539.

66. Zhu X, Zhao Z, Feng Y, Li P, Liu F, Liu J, et al. Genetic diversity of the Plasmodium falciparum apical membrane antigen I gene in parasite population from the China-Myanmar border area. Infect Genet Evol. 2016:39:155-62.

67. Su X, Ferdig MT, Huang Y, Huynh CQ, Liu A, You J, et al. A genetic map and recombination parameters of the human malaria parasite Plasmodium falciparum. Science. 1999;286:1351-3.

68. Anderson TJ, Haubold B, Williams JT, Estrada-Franco JG, Richardson L, Mollinedo $R$, et al. Microsatellite markers reveal a spectrum of population structures in the malaria parasite Plasmodium falciparum. Mol Biol Evol. 2000;17:1467-82

69. Gentil F, Bargieri DY, Leite JA, Francoso KS, Patricio MB, Espindola NM, et al. A recombinant vaccine based on domain II of Plasmodium vivax Apical Membrane Antigen 1 induces high antibody titres in mice. Vaccine. 2010:28:6183-90.

70. Dutta S, Lee SY, Batchelor AH, Lanar DE. Structural basis of antigenic escape of a malaria vaccine candidate. Proc Natl Acad Sci U S A. 2007:104:12488-93.

71. Takala SL, Coulibaly D, Thera MA, Batchelor AH, Cummings MP, Escalante AA, et al. Extreme polymorphism in a vaccine antigen and risk of clinical malaria: implications for vaccine development. Sci Transl Med. 2009;1:2ra5.

\section{Submit your next manuscript to BioMed Central and we will help you at every step:}

- We accept pre-submission inquiries

- Our selector tool helps you to find the most relevant journal

- We provide round the clock customer support

- Convenient online submission

- Thorough peer review

- Inclusion in PubMed and all major indexing services

- Maximum visibility for your research

Submit your manuscript at www.biomedcentral.com/submit

) Biomed Central 